\title{
PERIPHERAL ACYCLICITY IN 3-MANIFOLDS
}

\author{
DUŠAN REPOVŠ \\ (Received 3 April 1985; revised 15 November 1985) \\ Communicated by J. H. Rubinstein
}

\begin{abstract}
We study weak and strong peripheral 1-acyclicity, a homology version of D. R. McMillan, Jr.'s weak cellularity criterion and cellularity criterion, for embeddings of compacta in 3-manifolds. In contrast with the two cellularity criteria we prove that the two peripheral acyclicities are equivalent and moreover, for compacta of dimension at most 1, independent of the embedding. We also give some results concerning regular neighborhoods of compact polyhedra in 3-manifolds.
\end{abstract}

1980 Mathematics subject classification (Amer. Math. Soc.): 57 N 60, 57 N 40, 57 Q 40.

\section{Introduction}

Let $K$ be a subset of an ANR $X$. We say that the inclusion $K \subset X$ satisfies the weak cellularity criterion $(W C C)$ (respectively, cellularity criterion $(C C)$ ) if for each neighborhood $U \subset X$ of $K$ there exists a neighborhood $V \subset U$ of $K$ such that any loop in $V-K$ is contractible in $U$ (respectively, $U-K$ ). We say that $K$ has property 1-UV if for each neighborhood $U \subset X$ of $K$ there exists a neighborhood $V \subset U$ of $K$ such that any loop in $V$ is contractible in $U$. Conditions WCC and CC are concepts due to D. R. McMillan, Jr. [7], [8], while property 1-UV has been studied by many; see for example [5].

In this paper we shall study homology versions of these three loop conditions. So let $K$ be a subset of an ANR $X$ and let $R$ be a principal ideal domain (PID). We say that the inclusion $K \subset X$ is strongly (respectively, weakly peripherally,

(C) 1987 Australian Mathematical Society $0263-6115 / 87 \$ A 2.00+0.00$ 
strongly peripherally) 1-acyclic over $R$ (SA, (respectively, WPA, SPA)) if, for each neighborhood $U \subset X$ of $K$, there exists a neighborhood $V \subset U$ of $K$ such that the inclusion-induced homomorphism $H_{1}(V ; R) \rightarrow H_{1}(U ; R)$ (respectively, $\left.H_{1}(V-K ; R) \rightarrow H_{1}(U ; R), H_{1}(V-K ; R) \rightarrow H_{1}(U-K ; R)\right)$ is trivial.

It is well known that SA does not depend upon the embedding of $K$ into $X$ (provided $X$ is an ANR) and that, furthermore, for $R$ a field, SA is equivalent to the condition $\check{H}^{1}(K ; R) \cong 0\left[5\right.$, Proposition 2.2], where $\check{H}^{*}(-; R)$ is the Čech cohomology with coefficients in $R$. The following example due to R. C. Lacher [4] shows that WPA and SPA may depend upon the embedding. Let $X=S^{2} \times S^{1}$, let $K=S^{2} \vee S^{1}$, and let $\varphi: K \rightarrow \mathbb{R}^{3}$ and $\psi: \mathbb{R}^{3} \rightarrow X$ be arbitrary PL embeddings. Then $K \subset X$ is SPA over every PID $R$ (since $X-K$ is an open 3-cell) while $(\psi \circ \varphi)(K) \subset X$ is not even WPA over any PID $R$ (just let $U=\psi\left(\mathbb{R}^{3}\right)$ ). It is not a coincidence that $\operatorname{dim} K=2$ in our example, for we shall prove that, for $\operatorname{dim} K \leqslant 1$ and $X$ an $R$-orientable 3-manifold, all three acyclicities are equivalent, and hence, in particular, independent of the embedding (Theorem 2.3). This result is an analogue of [4, p. 139], where it is shown that if $K$ is a compact subset of a PL $n$-manifold $M$ of codimension at least $2, n \neq 4$ (this condition can now be omitted by [1]), then WCC is equivalent to the property 1-UV, and thus independent of the embedding [5, Proposition 2.1].

Another result is Theorem 2.1, which asserts that in a 3-manifold SPA is the same as WPA (over $\mathbf{Z}_{2}$ or $\mathbf{Z}$ ). This is in contrast with the fact that WCC is a strictly weaker property than CC (just consider any noncellular arc).

In 3-manifolds, compacta which are WPA $\left(\mathbf{Z}_{2}\right)$ have some nice geometric properties, for example (i) in a nonorientable closed 3-manifold $M$ there is an upper bound on the number of pairwise disjoint compacta that fail to have a neighborhood in $M$ embeddable in $\mathbb{R}^{3}$, provided these compacta are $\operatorname{WPA}\left(\mathbf{Z}_{2}\right)$ embedded in $M$ (this extends T. E. Knoblauch's finiteness theorem [3] to nonorientable 3-manifolds) [9, Proposition 2.1]; and (ii) in 3-manifolds every WPA $\left(\mathbf{Z}_{2}\right)$ embedded compact connected set $K$ which doesn't separate its connected neighborhoods has arbitrarily small neighborhoods of the type $Q+$ (orientable 1-handles), where the compact 3-manifold $Q$ with $\partial Q=S^{2}$ captures the "nonorientability" of $K$, while the handles describe the "wildness" of the embedding of $K$ into $M$ [9, Proposition 2.2].

As an application of these results one can improve acyclic maps on 3-manifolds to yield cell-like resolutions [9, Theorem 1.1].

Our investigations of the three peripheral acyclicities were inspired by our joint work with R. C. Lacher on acyclic images of nonorientable 3-manifolds [9] and also by the following question (to our knowledge still open) raised by Lacher in 1975: suppose that $f: K \rightarrow N$ is an embedding of a compact set $K$ into an $n$-manifold $N, f$ homotopic to the inclusion $K \subset N$, and suppose that $K \subset N$ 
has WCC. Does $f(K) \subset N$ also have WCC [4, p. 139]?. In this paper we shall give an affirmative answer for the case when $K$ is a polyhedron, $N$ is a 3-manifold, and $f$ is PL (Theorem 3.1).

\section{Relations between WPA, SPA, and SA}

It is clear that SPA implies WPA. We show that for compacta in 3-manifolds the two peripheral acyclicities are equivalent over $\mathbf{Z}_{2}$ and $\mathbf{Z}$.

THEOREM 2.1. Let $K$ be a compact set in the interior of a 3-manifold $M$. Suppose that $K \subset M$ is WPA over $\mathbf{Z}_{2}$ (respectively, over $\mathbf{Z}$ ). Then $K \subset M$ is SPA over $\mathbf{Z}_{2}$ (respectively, over $\mathbf{Z}$ ).

Proof. We shall supress the coefficients. Using the hypothesis, we can express $K$ as the intersection of compact 3-manifolds $N_{i} \subset$ int $M$ with boundary such that for each $i, N_{i+1} \subset$ int $N_{i}$, and for which the inclusion-induced homomorphisms $H_{1}\left(N_{i}-K\right) \rightarrow H_{1}\left(N_{i-1}\right)$ are trivial. We shall show that by choosing an appropriate subsequence of $\left\{N_{i}\right\}$, each homomorphism $H_{1}\left(N_{i}-K\right) \rightarrow$ $H_{1}\left(N_{i-1}-K\right)$ is trivial, too.

Let $\alpha$ be a simple closed curve in $N_{i}-K$. Then there is an integer $j>i$ such that $\alpha \subset N_{i}-N_{j}$. Let $\Sigma \subset \partial N_{j+1}$ be a component of $\partial N_{j+1}$. Since $\Sigma$ is a closed 2-manifold, it contains a bouquet $T$ of finitely many simple closed curves such that $\Sigma-T$ is a union of pairwise disjoint open 2-cells. Let $\beta \subset T$ be one of these loops. Since $H_{1}\left(\partial N_{j+1}\right) \rightarrow H_{1}\left(N_{j}\right)$ is trivial, $\beta$ bounds a surface $\Gamma_{\beta}$ in $N_{j}$. Also, $\alpha$ bounds a surface $\Gamma_{\alpha}$ in $N_{i-1}$, since $H_{1}\left(N_{i}-K\right) \rightarrow H_{1}\left(N_{i-1}\right)$ is trivial. Put the surfaces $\Gamma_{\alpha}$ and $\Gamma_{\beta}$ in general position. Then $\Gamma_{\alpha} \cap \beta=\left\{p_{1}, \ldots, p_{t}\right\}$ for some points $p_{i} \in \beta$. We may assume that for each $i, p_{i}$ lies between $p_{i-1}$ and $p_{i+1}$ on $\beta$ (where $p_{t+1}=p_{1}$ ). Note also that each $p_{i}$ lies in int $\Gamma_{\alpha}$ because $\beta \cap \partial \Gamma_{\alpha}=\beta \cap$ $\alpha=\varnothing$.

We now show how to modify $\Gamma_{\alpha}$ so that it will miss $\beta$. Consider $X=\Gamma_{\alpha} \cap \Gamma_{\beta}$. Then $X$ is a compact (possibly disconnected) 1-manifold with (possibly nonempty) boundary, $\partial X \subset \partial \Gamma_{\alpha} \cup \partial \Gamma_{\beta}$. Suppose $t=1$. Then one of the components of $X$ must be an $\operatorname{arc} A$ (while others are then necessarily simple closed curves in int $\Gamma_{\beta}$ ). Let $\partial A=\{p, q\}$. Then, say, $\{p\}=\Gamma_{\alpha} \cap \beta$. Now, $q \notin \partial \Gamma_{\alpha}$ because $\Gamma_{\beta} \cap \partial \Gamma_{\alpha}=\Gamma_{\beta} \cap \alpha=\varnothing$, since $\Gamma_{\beta} \subset N_{j}$ and $\alpha \subset N_{i}-N_{j}$. On the other hand, $q \notin \partial \Gamma_{\beta}$ because $\left(\partial \Gamma_{\beta}-\{p\}\right) \cap \Gamma_{\alpha}=\left(\Gamma_{\alpha} \cap \beta\right)-\left\{p_{1}\right\}=\varnothing$. Therefore, $q \notin$ $\partial \Gamma_{\alpha} \cup \partial \Gamma_{\beta}$. This contradiction shows that $t=1$ is impossible.

Suppose $t=2$. Change $\Gamma_{\alpha}$ to $\Gamma_{\alpha}^{*}$ by removing the interiors of two small disjoint disks $D_{1}$ and $D_{2}$ in int $\Gamma_{\alpha}$, centered at $p_{1}$ and $p_{2}$, respectively, and by adding to $\Gamma_{\alpha}-\operatorname{int}\left(D_{1} \cup D_{2}\right)$ an annulus $A$ from the boundary of a small closed 
regular neighborhood of $\beta$ such that $\partial A=\partial\left(D_{1} \cup D_{2}\right)$. Clearly, $\beta \cap \Gamma_{\alpha}^{*}=\varnothing$, where $\Gamma_{\alpha}^{*}=\left(\Gamma_{\alpha}-\operatorname{int}\left(D_{1} \cup D_{2}\right)\right) \cup A$ is the new $\Gamma_{\alpha}$.

At this point we make a remark concerning the orientability of $\Gamma_{\alpha}^{*}$ if $R=Z$ Z. In this case we may assume that $\Gamma_{\alpha}$ and $\Gamma_{\beta}$ were orientable from the start. Since $\alpha$ and $\beta$ don't link, the (integral) intersection number of $\Gamma_{\alpha}$ and $\beta$ must be zero. Therefore, if we choose orientations for $\Gamma_{\alpha}$ and $\Gamma_{\beta}$, we can assign an index $\varepsilon_{i}=1$ or -1 to each point of intersection $p_{i}$. We may then choose the arcs of $\beta$ along which we perform the described surgery in such a way that they connect adjacent points $p_{i}$ and $p_{j}$ (i.e. $|i-j|=1$ ) with opposite indices: $\varepsilon_{i} \cdot \varepsilon_{j}=-1$. In this case $\Gamma_{\alpha}^{*}$ will always remain orientable.

Another remark concerns other loops of the bouquet $T$ : we may always choose not to do the surgery along subarcs of $\beta$ which run through the "wedge" point of $T$ (i.e. the only point of $T$ that all loops have in common). Therefore, when we begin to disentangle $\Gamma_{\alpha}$ from $\beta^{\prime} \neq \beta$ (after we have made $\Gamma_{\alpha}$ disjoint from $\beta$ ), we never introduce intersections of $\Gamma_{\alpha}$ with $\beta$.

If $t>2$, then we can perform the preceding operation on all pairs $\left(p_{2 i-1}, p_{2 i}\right)$, $1 \leqslant i \leqslant[t / 2]$. The argument we gave to dismiss the case $t=1$ shows that $t$ must be an even number, so this process yields a surface $\Gamma_{\alpha}^{*}$ disjoint from $\beta$.

It follows that $\alpha$ is null homologous in the complement of $\beta$. Repeating this, if necessary, we can find an $R$-orientable surface $\Gamma$ in $N_{i-1}$ such that $\alpha=\partial \Gamma$ and $\Gamma \cap(U\{\beta \mid \beta \in T\})=\varnothing$. Thus, if $\Gamma$ hits $N_{j+1}$ at all, it enters through open disks in $\partial N_{j+1}$, and so it can be cut off at $\partial N_{j+1}$. Hence $\alpha$ is already null-homologous over $R$ in $N_{i}-N_{j+1} \subset N_{i}-K$.

THEOREM 2.2. Let $R$ be a PID and let $K$ be a compact set in the interior of an $R$-orientable 3-manifold $M$. Suppose that $K$ is $\operatorname{SA}(R)$. Then $K \subset M$ is $\operatorname{SPA}(R)$.

Proof. We shall supress the coefficients. Let $V \subset U \subset M$ be neighborhoods of $K$ such that the inclusion-induced homomorphism $H_{1}(V) \rightarrow H_{1}(U)$ is trivial. Consider the following commutative diagram

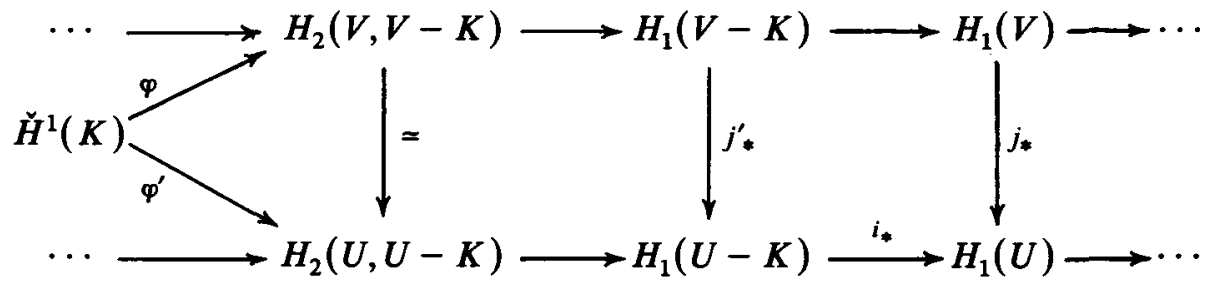

where the horizontal sequences are from the homology sequences of the pairs $(V, V-K)$ and $(U, U-K)$, respectively, and where $\varphi, \varphi^{\prime}$ are duality isomorphisms [11, Theorem 6.2.17]. Now, by [5, Proposition 2.2], $\check{H}^{1}(K)=0$; hence $i_{*}$ is a monomorphism. By hypothesis, $j_{*}=0$; hence $j_{*}^{\prime}=0$. 
Note that the converse of Theorem 2.2 is false: let $M=S^{2} \times S^{1}$ and let $K=S^{2} \vee S^{1}$. Then $M / K=S^{3}$, so $K$ is SPA over every PID $R$. On the other hand, $K$ is clearly not SA over any $R$. In this example $\operatorname{dim} K=2$. The next theorem shows that there can be no counterexample with $\operatorname{dim} K \leqslant 1$.

THeOREM 2.3. Let $R$ be a PID and let $K$ be a compact set in the interior of an $R$-orientable 3-manifold $M$. Suppose that $\operatorname{dim} K \leqslant 1$. Then the following statements are equivalent:

(i) $K$ is $\mathrm{SA}(R)$;

(ii) $K$ is $\operatorname{SPA}(R)$;

(iii) $K$ is $\operatorname{WPA}(R)$.

Proof. We shall supress the coefficients. The implication (i) $\Rightarrow$ (ii) follows by Theorem 2.2, while (ii) $\Rightarrow$ (iii) is obvious. We prove (iii) $\Rightarrow$ (i). Thus, let $V \subset U \subset$ $M$ be neighborhoods of $K$ such that the inclusion-induced homomorphism $H_{1}(V-K) \rightarrow H_{1}(U)$ is trivial. Let $\alpha$ be any 1-cycle in $V$. By [4, Lemma 2.1] $\alpha$ is homologous to a 1 -cycle $\beta \in Z_{1}(V-K)$. By hypothesis $\beta$ is null-homologous in $U$; hence so is $\alpha$.

THEOREM 2.4. Let $K$ be a compact set in the interior of a 3-manifold $M$. Then the following statements are equivalent ( for $R=\mathbf{Z}_{2}$ or $\mathbf{Z}$ ):

(i) $K \subset M$ is WPA( $R)$;

(ii) $K \subset M$ is $\operatorname{SPA}(R)$;

(iii) There exists a neighborhood $W \subset M$ of $K$ such that each simple closed curve in $W-K$ is $R$-homologous to zero in $M-K$.

REMARK 2.5. Let $W \subset M$ be an open neighborhood of $K$ as in (iii) above. Then by [8, Lemma 1] $K$ is $\mathrm{SA}(R)$ if and only if, in addition, each simple closed curve in $W$ is $R$-homologous to zero in $M$. This gives a good measure of the difference between the two acyclicities (over $R$ ).

Proof. We only need to prove (iii) $\Rightarrow$ (ii) (because we have (i) $\Rightarrow$ (ii) by Theorem 2.1, while the other two implications are clear). Let $U \subset M$ be a neighborhood of $K$. We may assume that $U \subset W$, that $U$ is a compact 3-manifold with boundary, and that $K \subset \operatorname{int} U$. Let $\Sigma \subset \partial U$ be a component of $\partial U$. Then there is a bouquet $T \subset \Sigma$ of simple closed curves such that $\Sigma-\cup\{\beta \mid \beta \in T\}$ is a union of pairwise disjoint open 2-cells. By hypothesis each curve $\beta \in T$ bounds an $R$-orientable surface $\Gamma_{\beta}$ in $M-K$. Let $V=\operatorname{int} U-U\left\{\Gamma_{\beta} \mid \beta \in T\right\}$ and let $\alpha$ be a simple closed curve in $V-K$. Then $\alpha$ bounds an $R$-orientable surface $\Gamma_{\alpha}$ in $M-K$. Using the same arguments as in the proof of Theorem (2.1), we can show 
that $\alpha$ bounds an $R$-orientable surface $\Gamma_{\alpha}^{*}$ in $M-(K \cup(\cup\{\beta \mid \beta \in T\}))$. Hence $\alpha$ enters $\Sigma$ through open disks and can thus be cut off at $\Sigma$. So we may assume that $\Gamma_{\alpha}^{*} \subset U-K$, i.e., that every 1-cycle in $V-K$ is null-homologous in $U-K$.

CoRollary 2.6 (D. R. McMillan, Jr. [8]). Let $K$ be a compact set in the interior of a 3-manifold $M$ and suppose that $H_{1}(M-K ; R)=0$, where $R=\mathbf{Z}_{2}$ or $\mathbf{Z}$. Then $K \subset M$ is $\operatorname{SPA}(R)$.

Proof. Apply Theorem 2.4 with $W=M$.

\section{Regular neighborhoods of compact polyhedra}

Throughout this section we work with $\mathbf{Z}_{2}$ or $\mathbf{Z}$ coefficients. We study regular neighborhoods of compact polyhedra in 3-manifolds. Our main result in this section is Theorem 3.1, which also answers in the affirmative a question of R. C. Lacher [4]: suppose that $f$ is a PL embedding of a compact polyhedron $K$ in a 3-manifold $M$ such that $f$ is homotopic to the inclusion $K \subset M$, and suppose that $K \subset M$ satisfies WCC; does $f(K) \subset M$ satisfy WCC? Note that by Theorem 2.1, WPA = SPA for the class of compacta in 3-manifolds, so we may drop the adverbs "weakly" and "strongly".

Theorem 3.1. Suppose that $f: K \rightarrow M$ is a PL embedding of a compact polyhedron $K$ in the interior of a 3-manifold $M$. Suppose that $f$ is homotopic to the inclusion $K \subset M$ and that $K \subset M$ is peripherically 1-acyclic or satisfies WCC. Then $f(K) \subset M$ is peripherically 1-acyclic and satisfies WCC.

We shall first prove two lemmas.

LEMMA 3.2. Let $f_{1}, f_{2}: K \rightarrow$ int $M$ be homotopic PL embeddings of a compact polyhedron $K$ in a PL m-manifold. Let $N_{i} \subset$ int $M$ be a regular neighborhood of $f_{i}(K)$ in $M$. Then $\partial N_{1}$ and $\partial N_{2}$ have the same Euler characteristics.

Proof. Let $r \neq 0$ be any even natural number satisfying $r \geqslant 2 k-m+3$, where $k=\operatorname{dim} K$ and $m=\operatorname{dim} M$. Choose a triangulation of $M \times \mathbb{R}^{r}$ consistent with the one on $M$ and define PL embeddings $F_{i}: K \rightarrow M \times \mathbb{R}^{r}$ by $F_{i}=f_{i} \times 0$, $i=1,2$. Since the $f_{i}$ 's are homotopic, there is a homotopy $H: K \times I \rightarrow M \times \mathbb{R}^{r}$ from $F_{1}$ to $F_{2}$. Define a map $H^{*}: K \times I \rightarrow M \times \mathbb{R}^{r} \times I$ by $H^{*}(x, t)=\left(H_{t}(x), t\right)$ for each $(x, t) \in K \times I$. We may assume that $H^{*}$ is PL. Since $2 k-m+2<r$, 
it follows that $2(k+1)-(m+r+1)<2 k+2-m-(2 k-m+2)-1=$ $-1<0$, so by general position we may assume that $H^{*}$ is a PL embedding and hence a concordance [2, p. 182]. Now $(m+r)-3 \geqslant m+(2 k-m+3)-3=$ $2 k \geqslant k$, so by [2, Corollary 1.4], $F_{1}$ and $F_{2}$ are ambient PL isotopic.

Let $N_{i}^{*}=N_{i} \times B^{r}, i=1,2$. Then by [10, Corollary 3.29], $N_{i}^{*}$ is a regular neighborhood of $F_{i}(K)$ in $M \times R^{r}$, so $N_{1}^{*} \cong{ }_{\mathrm{PL}} N_{2}^{*}$. In particular, $\partial N_{1}^{*} \cong \partial N_{2}^{*}$. Now, $\partial N_{i}^{*}=\left(\partial N_{i} \times B^{r}\right) \cup\left(N_{i} \times S^{r-1}\right)$, and $\left(\partial N_{i} \times B^{r}\right) \cap\left(N_{i} \times S^{r-1}\right)=$ $\partial N_{i} \times S^{r-1}$. Therefore the Euler characteristics of $\partial N_{i}^{*}$ and $\partial N_{i}$ are equal. Now we have

$$
\begin{aligned}
\chi\left(\partial N_{i}^{*}\right) & =\chi\left(\partial N_{i} \times B^{r}\right)+\chi\left(N_{i} \times S^{r-1}\right)-\chi\left(\partial N_{i} \times S^{r-1}\right) \\
& =\chi\left(\partial N_{i}\right)+\chi\left(N_{i}\right) \cdot \chi\left(S^{r-1}\right)-\chi\left(\partial N_{i}\right) \cdot \chi\left(S^{r-1}\right)=\chi\left(\partial N_{i}\right)
\end{aligned}
$$

The second equality follows by the product formula for the Euler characteristic, and the third equality by the fact that $r$ was chosen to be even (hence $\chi\left(S^{r-1}\right)$ $=0)$. Consequently $\chi\left(\partial N_{1}\right)=\chi\left(\partial N_{1}^{*}\right)=\chi\left(\partial N_{2}^{*}\right)=\chi\left(\partial N_{2}\right)$.

LEMMA 3.3. Let $K$ be a compact polyhedron in the interior of a 3-manifold $M$. Then the following statements are equivalent

(i) $K \subset M$ is peripherally 1-acyclic;

(ii) $K \subset M$ satisfies WCC;

(iii) for every regular neighborhood $N \subset M$ of $K, \partial N$ is a collection of 2-spheres.

Proof. (i) $\Rightarrow$ (iii). Let $N \subset M$ be a regular neighborhood of $K$, where $K \subset M$ is peripherally 1-acyclic. Then there exists a regular neighborhood $N^{*} \subset$ int $N$ of $K$ in $M$ such that the inclusion-induced homomorphism $H_{1}\left(N^{*}-K\right) \rightarrow H_{1}(N)$ is trivial; hence so is $H_{1}\left(\partial N^{*}\right) \rightarrow H_{1}\left(N^{*}\right)$, since $\overline{N-N^{*}}=\partial N^{*} \times I$, and $N^{*}-K=\partial N^{*} \times[0,1) \simeq \partial N^{*}$. Consider the following exact homology sequence (over $\mathbf{Z}_{2}$ ) for the pair $\left(N^{*}, \partial N^{*}\right)$ :

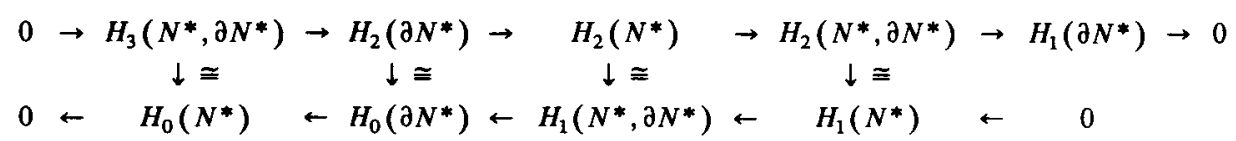

where the vertical isomorphisms are valid by Poincaré duality plus the universal coefficients theorem. Due to the exactness, the alternating sum of the ranks is zero; hence rank $H_{1}\left(\partial N^{*}\right)=0$. Since by [6, Lemma 4.1] $\partial N^{*}$ is orientable, it follows that $\partial N^{*}$ is a collection of 2-spheres.

(iii) $\Rightarrow$ (ii). This is clear. 
(ii) $\Rightarrow$ (i). Let $V \subset U \subset M$ be neighborhoods of $K$ such that the inclusion $i$ : $V-K \rightarrow U$ induces a zero homomorphism $i_{\#}$ on the fundamental groups. Consider the following commutative diagram

$$
\begin{array}{ccc}
\Pi_{1}(V-K) & \stackrel{i_{*}}{\rightarrow} & \Pi_{1}(U) \\
\downarrow h & & \downarrow h^{\prime} \\
H_{1}(V-K) & \stackrel{i_{*}}{\rightarrow} & H_{1}(U)
\end{array}
$$

where $h$ and $h^{\prime}$ are the Hurewicz epimorphisms. Since $i_{\#}=0$, it follows that $i_{*}=0$, too.

Proof of Theorem 3.1. We may assume that $K$ and $M$ are connected. Let $N \subset$ int $M$ be a regular neighborhood of $K$ and $N^{*} \subset$ int $M$ a regular neighborhood of $f(K)$. By [6, Lemma 4.1], $\partial N$ is orientable; hence, by Lemma 3.3, $\partial N$ is a collection of $n$ 2-spheres for some $n$. Therefore, by Lemma 3.2, $\chi\left(\partial N^{*}\right)=$ $\chi(\partial N)=2 n$. According to Lemma 3.3, the proof will be completed as soon as we demonstrate that the following assertion holds.

ASSERTION. $\partial N^{*}$ consists of $n$ 2-spheres.

Proof. We shall work with $\mathbf{Z}_{2}$ coefficients. Consider the Mayer-Vietoris sequence of the triple $(M, N, \overline{M-N})$ :

$$
0 \rightarrow H_{3}(M) \stackrel{\Delta}{\rightarrow} H_{2}(\partial N) \stackrel{f}{\rightarrow} H_{2}(N) \oplus H_{2}(\overline{M-N}) \stackrel{g}{\rightarrow} H_{2}(M) \rightarrow \cdots
$$

Then $g=g_{1}+g_{2}$, where $g_{1}: H_{2}(N) \rightarrow H_{2}(M)$ and $g_{2}: H_{2}(\overline{M-N}) \rightarrow H_{2}(M)$ are the inclusion-induced homomorphisms [11, Chapter 4.6]. Clearly,

$$
\operatorname{dim} H_{2}(\partial N)=1+\operatorname{dim}(\operatorname{Ker} g) .
$$

Let $A=\left\{x \in H_{2}(M) \mid x \cdot i(y)=0\right.$ for every $\left.y \in H_{1}(K)\right\}$, where $\cdot$ is the $(\bmod 2)$ intersection number, and where $i: H_{1}(K) \rightarrow H_{1}(M)$ is the inclusion-induced homomorphism.

Consider the following diagram

$$
\begin{aligned}
& \cdots H_{3}(M, M-K) \stackrel{\Delta}{\rightarrow} H_{2}(M-K) \stackrel{\jmath}{\rightarrow} \quad H_{2}(M) \stackrel{k}{\rightarrow} \quad H_{2}(M, M-K) \\
& \equiv \downarrow \varphi \\
& \tilde{H}^{0}(K) \\
& \cong \downarrow \varphi \\
& \check{H}^{1}(K)
\end{aligned}
$$


where the horizontal line represents the homology sequence of the pair ( $M, M-$ $K$ ), and where $\varphi$ is the duality isomorphism [11, Theorem 6.2.17]. Since $\Delta=0$, it follows that $j$ is one-to-one; hence $H_{2}(M-K)$ can be naturally identified with $\operatorname{Ker}\left[H_{2}(M) \rightarrow H_{2}(M, M-K)\right]=\operatorname{Ker}\left[H_{2}(M) \rightarrow \check{H}^{1}(K)\right]=A$. Consequently, Im $g_{2}=A$, and we conclude that

$$
\mathrm{g}_{2}: \mathrm{H}_{2}(\overline{M-N}) \rightarrow A \subset H_{2}(M) \text { is one-to-one. }
$$

Define now a map $\alpha$ : $\operatorname{Ker} g \rightarrow g_{1}^{-1}(A)$ by $\alpha\left(a_{1}, a_{2}\right)=a_{1}$, for every $\left(a_{1}, a_{2}\right) \in$ Ker $g$. Then $\alpha$ is surjective: Let $a_{1} \in g_{1}^{-1}(A)$. Then $g_{1}\left(a_{1}\right) \in A=\operatorname{Im} g_{2}$ (by (2)). Hence $g_{1}\left(a_{1}\right)=g_{2}\left(a_{2}\right)$ for some $a_{2} \in H_{2}(\overline{M-N})$, and so $g\left(a_{1}, a_{2}\right)=g_{1}\left(a_{1}\right)+$ $g_{2}\left(a_{2}\right)=2 \cdot g_{1}\left(a_{1}\right)=0$. Thus $\left(a_{1}, a_{2}\right) \in \operatorname{Ker} g$. Also, $\alpha$ is injective: suppose that $\alpha\left(a_{1}, a_{2}\right)=0$ for some $\left(a_{1}, a_{2}\right) \in \operatorname{Ker} g$. Then $a_{1}=0$. Hence $g_{2}\left(a_{2}\right)=g_{1}\left(a_{1}\right)=$ $g_{1}(0)=0$, and so by (2), $a_{2}=0$.

Therefore $\alpha$ is an isomorphism, and we have the following relationship:

$$
\operatorname{dim}(\operatorname{Ker} g)=\operatorname{dim} g_{1}^{-1}(A) \text {. }
$$

Now $g_{1}$ and $A$ are obviously invariant under homotopic reembeddings of $K$ into $M$; hence $\operatorname{dim}(\operatorname{Ker} g)$ is invariant as well. Therefore, by $(1), \operatorname{dim} H_{2}(\partial N)=$ $\operatorname{dim} H_{2}\left(\partial N^{*}\right)$, and so, in particular, $N$ and $N^{*}$ must have the same number of boundary components. This proves our assertion and hence completes the proof of the theorem.

\section{Acknowledgements}

This work was completed during my stay at the Institut des Hautes Études Scientifiques (Bures-sur-Yvette, France) in 1984. I wish to acknowledge J. Cerf for making this visit possible. I should also like to thank F. Bonahon, and the referee for their helpful remarks.

\section{References}

[1] M. H. Freedman, 'The topology of four-dimensional manifolds', J. Differential Geom. 17 (1982), 357-453.

[2] J. F. P. Hudson, 'Concordance, isotopy, and diffeotopy', Ann. of Math . (2) 91 (1970), 425-448.

[3] T. E. Knoblauch, 'Imbedding compact 3-manifolds in $E^{3}$, Proc. Amer. Math. Soc. 48 (1975), 447-453.

[4] R. C. Lacher, 'A cellularity criterion based on codimension', Glasnik Mat. (2) 11 (1976), 135-140.

[5] R. C. Lacher, 'Cell-like mappings and their generalizations', Bull. Amer. Math. Soc. 83 (1977), 495-552. 
[6] R. C. Lacher and D. R. McMillan, Jr., 'Partially acyclic mappings between manifolds', Amer. J. Math. 94 (1972), 246-266.

[7] D. R. McMillan, Jr., 'A criterion for cellularity in a manifold', Ann. of Math. (2) 79 (1964), 327-337.

[8] D. R. McMillan, Jr., 'Acyclicity in three-manifolds', Bull. Amer. Math. Soc. 76 (1970), 942-964.

[9] D. Repovš and R. C. Lacher, 'Resolving acyclic images of nonorientable three-manifolds', Proc. Amer. Math. Soc. 90 (1984), 157-161.

[10] C. P. Rourke and B. J. Sanderson, Introduction to Piecewise-Linear Topology (Ergeb. der Math. Vol. 69, Springer-Verlag, Berlin, Heidelberg, New York, 1972).

[11] E. H. Spanier, Algebraic Topology (McGraw-Hill, New York, 1966).

\section{Department of Mathematics}

University of Texas

Austin, Texas 78712

U. S. A. 